\title{
ELECTRO-OPTICAL KERR EFFECT MEASUREMENTS IN CONDUCTING SYSTEMS
}

\author{
O.V. Prezhdo $a$, M.V. Khaschina ${ }^{b}$, Yu.P. Novikov ${ }^{b}$ and V.V. Prezhdo ${ }^{b, c}$ \\ ${ }^{a}$ Department of Chemistry, University of Washington, Seattle, WA 98115, USA \\ ${ }^{b}$ Department of Organic Chemistry, Kharkov State Polytechnic University \\ 21. Frunze Street, 310002 Kharkov, Ukraine \\ ${ }^{c}$ Institute of Chemistry, Pedagogical University, Chęcinska 5, 25-020 Kielce, Poland
}

(Received March 29, 1999; in final form June 16, 1999)

\begin{abstract}
A new method of investigation of electro-optical properties of conducting media is proposed based on a contactless scheme for capacitance measurements. Using alternating electric current, the method is applied to study Kerr effect in a series of single and multi-component conducting liquid systems.
\end{abstract}

PACS numbers: $35.20 . \mathrm{My}, 31.70 . \mathrm{Dk}$

\section{Introduction}

Electro-optical Kerr effect is a popular tool of experimental physical chemistry [1-8]. In recent years, an increased interest has been drawn to the electro-optical properties of associated liquid systems. The associated systems containing water are ubiquitous in nature and attract particular attention. In present, the Kerr constants of many non-polar and slightly polar dielectrics are well known. The water based systems, on the other hand, are studied insufficiently, due to the abnormally high conductivities that are observed in aqueous solutions and complicate the measurements.

The application of traditional approaches to investigation of electro-optical systems meets two profound difficulties: (1) the difficulty of proper measurement of Kerr effect of conducting media, and (2) the instability of many systems subject. to strong electric fields.

The existing experimental techniques for Kerr effect measurements can be separated into two categories based on the electric field that is used to induce birefringence, namely, the techniques that use constant electric fields and the ones that use alternating electric fields. The traditional techniques for measurement of electro-optical constants in a constant field are suitable for good dielectrics with specific resistances of no less than $10^{10} \Omega \mathrm{cm}$. The constant electric field techniques 
are not applicable to conducting systems. The problem with conducting systems arises due to the difficulty of accurate determination of the strength of the field inside the system. In addition, a constant electric field induces convection currents in the conducting system, therewith perturbing chemical equilibria.

The techniques based on alternating electric fields can be more suitable for measurements of the Kerr and higher order nonlinear electro-optical effects. Alternating currents limit the timescale of the perturbation, eliminating problems due to orientation relaxation of molecules. On the other hand, alternating fields usually have to be strong and, therefore, cannot be used with the systems that exhibit weak intermolecular interactions and are unstable in strong fields. The use of moderate and low-frequency alternating fields for studies of electro-optical properties of chemical systems has not been fully explored yet. Such fields have potential to circumvent the difficulties with constant and high-frequency alternating fields, leading to more accurate results for certain types of chemical systems [6].

The aim of the present paper is the development of a novel techuique of the Kerr effect measurements in conducting systems using a low-frequency electric field. The technique is implemented with the apparatus that was developed earlier and is described in Ref. [6].

Conductivity in a system under study leads to a conductivity current in the Kerr cell. The conductivity current is mediated by jons and other mobile charge carriers. The presence of the conductivity current in the Kerr cell dramatically increases consumption of power. Systems heat up giving rise to convection currents. Convection depolarizes the light beam that is used for the Kerr effect measurements. In addition, mobile charge carriers form the Helmholtz double layer, destroying homogeneity of the electric field within the cell. The strength of the electric field between the electrodes becomes a nonlinear function of both the applied voltage [9] and system's conductivity [10-12]. The electric field becomes dependent on the geometry of the Kerr cell as well. These factors are present in the systems with high electric conductivity and deteriorate the results of the Kerr effect measurements in such systems.

The Kerr effect measurements that employ ultrashort electric pulses resolve some of the problems for the conducting systems [2, 3, 13-16]. The electric field is turned on only for a short interval so that the system heating is negligible. However, the electric fields that have to be used in the pulsed method are usually no less than $10^{4} \mathrm{~V} / \mathrm{cm}$. Ellis and Llewelin quoting the USA National Burea of Standards [17] state that the fields of this intensity do not provide the reliable Kerr constants for conducting systems such as water and aqueous solutions, even if electric pulses as short as $1 \mu$ s are used. The errors that are introduced in such measurements commonly exceed $10 \%$. The equipment is often expensive, cumbersome, and complex both to manufacture and to use [15, 18-21].

A new approach to studies of conducting systems is offered by the optical Kerr effect (OKE) measurements that are carried out by the electrodeless method, where the electric field is applied directly to the sample [22]. Note that the Kerr effect is very sensitive to the anisotropy of the molecular tensor of optical polarizability. The Kerr effect is also very seusitive to both permanent and induced electric dipole moments [23]. Hence, combined with the measurements of other 
characteristics including dielectric permittivity, light scattering, and molecular refraction, OKE permits the determination of molecular properties, geometries, and local structures of investigated media [24]. An excellent review of the application of OKE to pure liquids, liquid crystals, and plastic crystals can be found in Ref. [25]. For the principles and details of the setup and the experimental procedure see, for example, Refs. [26, 27].

OKE is analogous to the electric Kerr effect that is observed in static and slowly varying fields. There are, nevertheless, significant differences between the two effects. The frequency of the optical electric field is so high that the field does not affect orientations of permanent molecular dipoles. Only induced dipole moments are important in OKE. In particular. the induced dipole moments determine the anisotropic part of the Keerr constant. Due to the high frequency of the applied electric field, OKE is extremely suitable for studies of aqueous solutions and other conducting systems. Ionic conductivity is absent in OKE, and the spatial charge distribution, the double layer, is too slow to be formed. The magnitude of OKE is only linearly dependent on temperature. Therefore, OKE can be used to separate the linear term and to study temperature nonlinearities of the static electric Kerr effect.

References $[28,29]$ describe a method of the Kerr effect measurements in conducting systems by means of alternating electric fields of small amplitudes. The scheme is reproduced in Fig. 1. The birefringence that takes place in the measurement cuvette in the presence of the alternating electric current of frequency $\omega_{1}$ is combined with the birefringence that takes place in the reference cuvette in the presence of the electric current of frequency $\omega_{2}$. The photocurrent containing the $\omega_{1}$ and $\omega_{2}$ signals on the top of the bacliground current reach the photodetector. The $\omega_{1}$ and $\omega_{2}$ signals pass through the filter, the photomultiplier and they are analyzed. From the comparison between the signals, the magnitude of the Kerr constant of the solution in the measurement cuvette is determined relative to the Kerr constant of the solution in the reference cuvette. In addition, the phase shift between the reference and measurement signals is obtained. The $\omega_{1} \neq \omega_{2}$ condition must be met in order that the two signals are separable. The scheme in Fig. 1 is based on reference measurements, rather than on the compensatory approach. The possibility to study conducting systems is achieved by the appropriate design of the

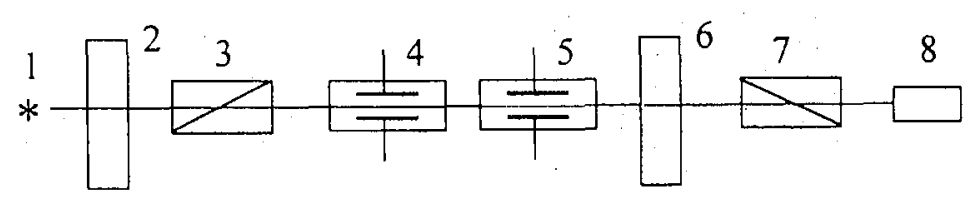

Fig. 1. Optical scheme for the Kerr effect measurements; light source (1); monochromator (2); polarizator (3); measurement cuvette (4); reference (compensating) cuvette (5); diffraction slit, $1 / 4$ of the optical wavelength wide (6); analyzer (7); photodetector and photomultiplier (8). 
photodetector and photomultiplier, rather than by the design of the measurement cuvette itself. The complications that are associated with the system conductivity and that have been discussed above are not eliminated, but only alleviated by the use of weak electric fields.

\section{Development of the contactless capacitance-based technique for the Kerr effect, measurements}

An alternative technique for the Kerr effect measurements of conducting systems can be suggested by malking an analogy with the contactless capacitance-based method of measurement of dielectric permittivity [30]. This method allows one to entirely eliminate the conductivity current in the measurement cuvette and to create a highly uniform electric field in the system, significantly increasing relia.bility and a.ccuracy of the measurements. A detailed analysis of the contactless capacitance-based method as well as details of design and manufacturing of the measurement cuvettes can be found in Refs. [31-33] for the dielectric permittivity and in Ref. [34] for the Kerr effect.

Let us describe a simple compensatory scheme for the measurement cuvette that is used for contactless determination of dielectric permittivities of liquids (Fig. 2). The electrodes are separated from the liquid by a layer of solid dielectric, chosen so that $R_{\mathrm{d}} \gg R_{\mathrm{l}}$, where $R_{\mathrm{d}}$ is the resistance of the dielectric and $R_{\mathrm{l}}$ is the resistance of the liquid. It is assumed that the frequency of the applied electric field $(\omega)$ is fixed. Then, the total conductivity between the electrodes 1 and 2 in the scheme equals

$$
J=\frac{\left[\omega^{2} c_{1} c_{2}+\omega^{2}\left(c_{1}+c_{2}\right) c_{2}\right] / R_{1}+j \omega\left[c_{2} / R_{1}+\omega^{2} c_{1} c_{2}\left(c_{1}+c_{2}\right)\right]}{1 / R_{1}^{2}+\omega^{2}\left(c_{1}+c_{2}\right)^{2}},
$$

where $j$ is the specific conductivity, and all other parameters are defined in Fig. 2. At the same time, for the scheme in Fig. 2 it is true that

$$
J=\frac{1}{R_{\mathrm{e}}}+j \omega c_{\mathrm{e}}
$$

where $R_{\mathrm{e}}$ and $c_{\mathrm{e}}$ are resistance and capacitance of the measurement cuvette.
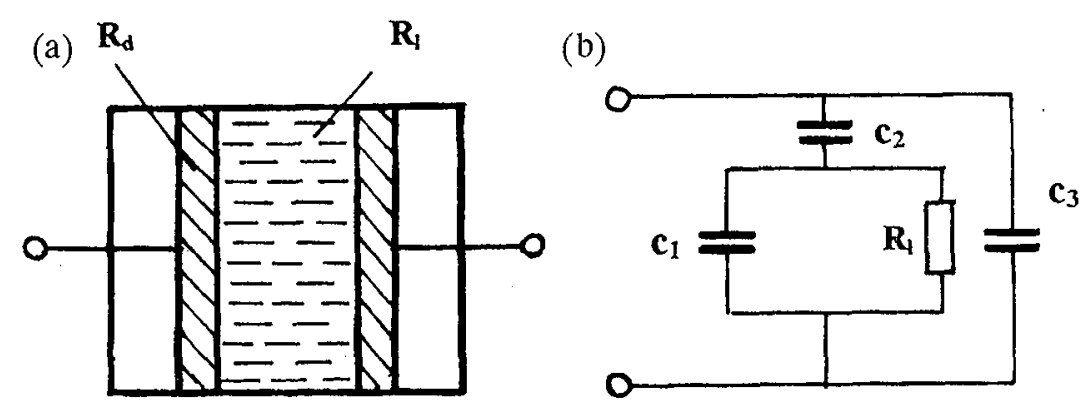

Fig. 2. Scheme of measurement cuvette with isolated electrodes (A) and its equivalent electronic scheme (B): $R_{\mathrm{d}}$ - dielectric resistance, $R_{\mathrm{l}}$ - liquid resistance, $c_{1}$ - capacity of liquid layer, $c_{2}$ - capacity of electrodes coverage; $c_{3}$ - parasitic capacity. 
Let us denote

$$
\frac{c_{1}}{c_{2}}+1=m, \quad \frac{1}{R \omega c_{2}}=n .
$$

Then

$$
J=\frac{n \omega c_{2}+j \omega\left[c_{2} n^{2}+m(m-1) c_{2}+\omega c_{3}\left(m^{2}+n^{2}\right)\right]}{m^{2}+n^{2}} .
$$

Comparing Eqs. (4) and (2) we obtain

$$
c_{e}=c_{2}+c_{3}-c_{2} \frac{m}{m^{2}+n^{2}}, \quad \frac{1}{R_{e}}=\omega c_{2} \frac{m}{m^{2}+n^{2}} .
$$

Since

$$
\frac{1}{R_{\mathrm{e}}}: c_{\mathrm{e}} \omega=\tan \delta_{\mathrm{e}}=k,
$$

where $\delta_{e}$ is the angle describing dielectric losses, the system of equations (5) can be transformed into

$$
c_{\mathrm{e}}=c_{2}+c_{3}-c_{2} \frac{m}{m^{2}+n^{2}}, \quad \frac{c_{\mathrm{e}}}{c_{2}} K=\frac{n}{m^{2}+n^{2}} .
$$

Solving for $m$ and $n$ and taking into account Eq. (3) we get

$$
c_{1}=\frac{c_{2}\left(c_{2}+c_{3}-c_{e}\right)\left(c_{e}-c_{3}\right)-c_{\mathrm{e}}{ }^{2} c_{2} K}{c_{\mathrm{e}}^{2} K^{2}+\left(c_{2}+c_{3}-c_{\mathrm{e}}\right)^{2}},
$$

which leads to the following criteria of applicability of the compensatory scheme

$$
\begin{aligned}
& c_{2}>\frac{c_{\mathrm{e}}^{2} K^{2}}{c_{\mathrm{e}}-c_{3}}-c_{3}+c_{\mathrm{e}}, \\
& R=\frac{\left(c_{2}+c_{3}-c_{\mathrm{e}}\right)^{2}+c_{\mathrm{e}}^{2} K^{2}}{K c_{2} c_{\mathrm{e}} \omega} .
\end{aligned}
$$

As follows from Eqs. (9) and (10), the applicability of the compensatory scheme of Fig. 2 depends on the parameters $c_{2}, c_{3}, c_{3}$, and $K=\tan \delta_{e}$.

The equivalence characteristics of the measurement cuvette $c_{e}$ and $\operatorname{tg} \delta_{e}$ were determined by the digital P-589 apparatus. The capacitance of the electrode $c_{2}$ was measured with the same apparatus for the cuvette filled with mercury in place of the solution uncler study. The capacitance $c_{3}$ was determined from calibration measurements performed with the standard liquids of known dielectric permittivities.

The capacitance of the dielectric layer of liquid can be calculated via Eq. (8). The dielectric permittivity of the liquid is calculated from

$$
c_{1}=\frac{\varepsilon \varepsilon_{0} s}{d} .
$$

The capacitance of the double layer at the electrode/solid dielectric interface is determined from the measurement of $c_{2}$ using the cuvette filled with mercury. The measurement also partially accounts for the capacitance of the dielectric/liquid interface double layer. The actual quantity measured is not just $c_{2}$, but the capacitance $c_{\mathrm{e}}^{\prime}=c_{2}^{\prime} c_{2} c_{2}^{\prime \prime} /\left(c_{2}^{\prime} c_{2}+c_{2}^{\prime \prime} c_{2}+c_{2}^{\prime} c_{2}^{\prime \prime}\right)$, where $c_{2}^{\prime}$ is the capacitance of the diffuse double layer at the electrode/solid dielectric interface, and $c_{2}^{\prime \prime}$ is the capacitance of the diffuse double layer at the solid dielectric/liquid interface. 
In the limiti of small $c_{3}$, i.e. when $c_{3} \rightarrow 0$, Eqs. (8) and (10) simplify to

$$
\begin{aligned}
& c_{1}=c_{2} c_{e} \frac{\left(c_{2}-c_{\mathrm{e}}\right)-K^{2} c_{\mathrm{e}}}{\left(c_{2}-c_{3}\right)^{2}+K^{2} c_{\mathrm{e}}^{2}}, \\
& R=\frac{\left(c_{2}-c_{\mathrm{e}}\right)^{2}+K^{2} c_{e}^{2}}{K c_{2} c_{e} \omega},
\end{aligned}
$$

while the strength of the electric field in the cuvette with the isolated electrodes becomes

$$
E^{2}=\left(\frac{U_{e}}{d_{e} c_{2}}\right)^{2}\left[\left(c_{2}+c_{3}-c_{e}\right)^{2}+c_{e}{ }^{2} K^{2}\right],
$$

where $d_{e}$ is the cuvette width.

Since $c_{2}=s \varepsilon \varepsilon_{0} / d$, it follows from the condition (9) that the width of the dielectric layer should be as small as possible, provided that the electrodes are properly isolated from the charge carriers in the liquid. The dielectric constant of the layer should be as large as possible. There exist several ways to decrease boundary effects for the electric field in the measurement cuvette, including optimization of the geometric shape of the electrodes and of the dielectric layer as well as development of special kinds of electrodes. This issue is discussed in detail in Refs. $[35,36]$.

Measurement cuvettes that are cylincler shaped are most convenient for contactless measurements of dielectric permitivities. The cylindrical shape of the cuvette significantly diminishes boundary effects in the electric field compared with rectangular cuvettes. In order to determine the optimal parameters of a cylindrical cuvette for contactless measurements of the Kerr constants of conducting systems, we have calculated the electric field inside the cuvette filled with electro-optically active dielectric as a function of geometric dimensions and dielectric permittivities of the cylinder and of the dielectric. The results obtained are based on the solutions to the Laplace equation for the infinite hollow cylinder placed in a uniform electric field and oriented perpendicular to the field (see Fig. 3). In this situation, the field $\left(E_{3}\right)$ inside the cylinder is also uniform and points along the applied field. The magnitude of the field inside the cylinder is given by

$$
E_{3}=\frac{4 \varepsilon_{1} \varepsilon_{2} E}{\left(\varepsilon_{1}+\varepsilon_{2}\right)\left(\varepsilon_{2}+\varepsilon_{3}\right)+\left(\varepsilon_{3}-\varepsilon_{2}\right)\left(\varepsilon_{2}-\varepsilon_{1}\right) r^{2} / R^{* 2}},
$$

where $E$ is the strength of the applied field; $\varepsilon_{1}, \varepsilon_{2}$, and $\varepsilon_{3}$ are the dielectric permittivities of the medium surrounding the cylinder, of the cylinder itself and of the electro-optically active dielectric, correspondingly; $r$ is the internal radius of the cylinder; $R^{*}$ is its external radius.

In order to determine the weakening of the uniform electric field after the cylinder is filled with the dielectric, we introduced the loss coefficient $\chi_{i}=E_{i} / E$, where $E_{i}$ is the strength of the field in the $i$-th zone, see Fig. 3. Figure 4 shows how the loss coefficient $\chi_{3}$ depends on the geometric dimensions and material of the cylinder, given $\varepsilon_{1}=1$ representing air, and $\varepsilon_{3}=36$ representing nitrobenzene. As can be seen in Fig. 4, the loss coefficient $\chi_{3}$ reaches the highest value of 18.5. 


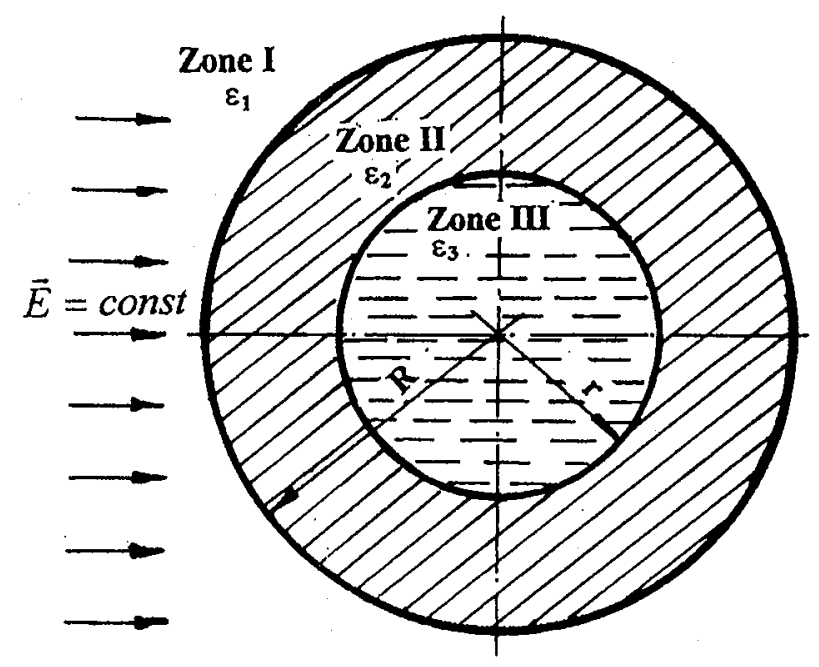

Fig. 3. Hollow dielectric cylinder in a uniform electric field.

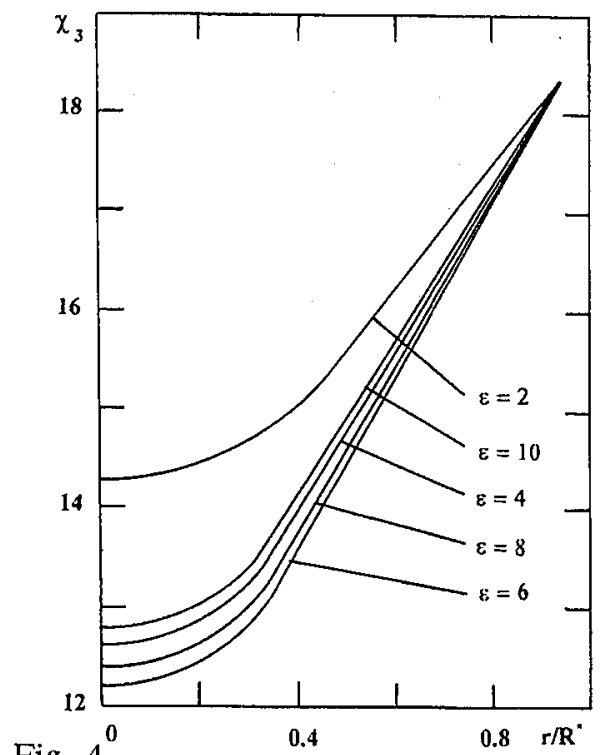

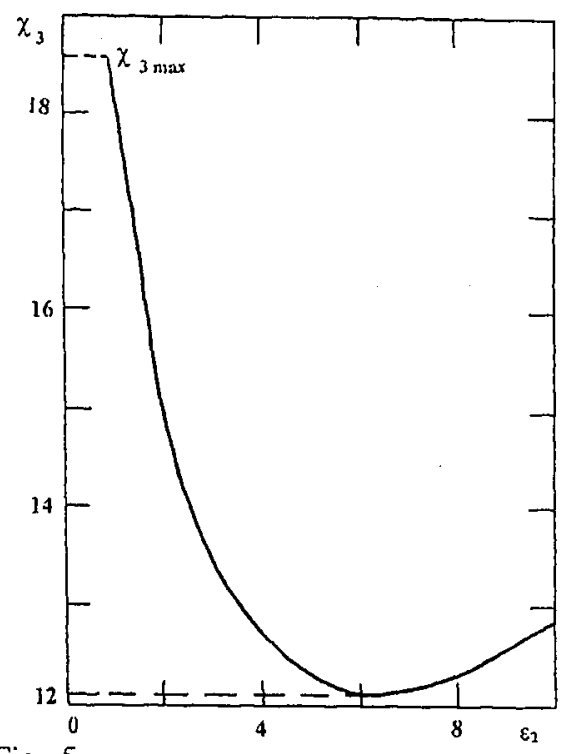

Fig. 5

Fig. 4. The dielectric loss coefficient $\chi_{3}$ as a function of the geometric parameter of the cylinder $r / R^{*}$ for several fixed values of $\varepsilon_{2}$.

Fig. 5. The dielectric loss coefficient $\chi_{3}$ as a function of the dielectric permittivity of the cylinder $\varepsilon_{2}$ for the measurement cuvette filled with nitrobenzene.

The coefficient is the smallest for porcelain $\left(\varepsilon_{2}=6\right)$ equaling 12.25 . The minimum of the loss coefficient is achieved, if either $\varepsilon_{2}=\sqrt{\varepsilon_{1} \varepsilon_{3}}$, or

$$
\chi_{\text {3min }}=\frac{1}{4 \varepsilon_{1}}\left[\left(\sqrt{\varepsilon_{1}}+\sqrt{\varepsilon_{2}}\right)^{2}+\frac{r^{2}}{R^{* 2}}\left(\sqrt{\varepsilon_{3}}-\sqrt{\varepsilon_{1}}\right)^{2}\right],
$$


which follows from Eq. (17)

$$
\chi_{3}=\frac{E_{3}}{E}=\frac{1}{4 \varepsilon_{1} \varepsilon_{2}}\left[\left(\varepsilon_{1}+\varepsilon_{2}\right)\left(\varepsilon_{2}+\varepsilon_{3}\right)+r^{2} R^{-2}\left(\varepsilon_{3}-\varepsilon_{2}\right)\left(\varepsilon_{2}-\varepsilon_{1}\right)\right] .
$$

Figure 5 shows the coefficient $\chi_{3}$ as a function of the dielectric permittivity of the cylinder $\varepsilon_{2}$, when $\varepsilon_{1}=1, \varepsilon_{3}=36$, and $r / R^{*} \rightarrow 0$. In this case

$$
\chi_{3}=\frac{1}{4}\left[\left(1+\sqrt{\varepsilon_{3}}\right)^{2}+\frac{r^{2}}{R^{* 2}}\left(\sqrt{\varepsilon_{2}}-1\right)^{2}\right] .
$$

The above analysis indicates that the cylindrical cuvettes for electro-optical measurements should be designed to have thick walls $\left(r / R^{*} \rightarrow 0\right)$. Then, the minimum of the loss coefficient will be achieved with $\varepsilon_{2}=\sqrt{\varepsilon_{3}}$.

The use of the contactless measurement cuvetites described above, both cylinclrical and rectangular in shape, allows us to measure the Kerr effect in conducting media without any modifications of the measurement apparatus itself. In contrast to the approach taken in Ref. [17], our approach takes advantage of the compensatory scheme, using both relative and absolute versions. As the result, the errors in the measurement data are greatly diminished.

\section{Results and discussion}

In order to study the Kerr effect in conducting media, we produced two rectangular glass cuvettes with isolated electrodes. The two rectangular cuvettes differed in the width of the liquid sample inside. We also developed a cylindrical porcelain cuvette with the following dimensions: $l=120 \mathrm{~mm}, R^{*}=20 \mathrm{~mm}$, $r=10 \mathrm{~mm}$.

The following relationship holds for the rectangular cuvettes [6]:

$$
\frac{B_{\lambda \text { ref }}}{B_{\lambda x}}=\text { const }_{0} \frac{U_{x}^{2}}{U_{\text {ref }}^{2}}
$$

where $B_{\lambda \text { ref }}$ and $B_{\lambda x}$ are the Kerr constants, whereas $U_{\text {ref }}$ and $U_{x}$ are electric fields applied to the reference material and to the system under investigation, respectively. The constant const 0 depends on the properties of the cuvette and is determined by calibration, using identical materials in both cuvettes. Considering $\mathrm{Eq}$. (14), the relationship of $\mathrm{Eq}$. (19) takes the form

$$
\left(\frac{U_{\text {ref }}}{d_{\text {ref }}^{*}}\right)^{2} /\left(\frac{U_{x}}{d_{x}^{*} c_{2}}\right)^{2}\left[\left(c_{2}+c_{3}-c_{e}\right)^{2}+c_{e}^{2} K^{2}\right]=\text { const }_{1} \frac{B_{\lambda x}}{B_{\lambda \text { ref }}} .
$$

The constant const ${ }_{1}$ is determined similarly to const $t_{0}$ by calibration with respect to a standard cuvette.

Since $d_{\text {ref }}^{* 2}, d_{x}^{* 2}$, and $c_{2}^{2}$ are the properties of the cuvette, we can incorporate them into the constant const ${ }_{2}$. Thus, we obtain the final expression for calculation of the Kerr effect constants from the results of measurements in cuvettes with isolated electrodes using the relative compensatory approach

$$
\frac{U_{\text {ref }}^{2}}{U_{x}^{2}\left[\left(c_{2}+c_{3}-c_{e}\right)^{2}+c_{e}^{2} K^{2}\right]}=\operatorname{const}_{2} \frac{B_{\lambda x}}{B_{\lambda \text { ref }}} \text {. }
$$

The phase shift that may occur between the electric fields applied to the reference and measurement cuvettes in such measurements was remedied by monitoring the phase of the entered signal in amplifier U-4-27. 
In the cases when $c_{3} \rightarrow 0$, Eq. (21) simplifies even further to give

$$
\frac{U_{\mathrm{rel}}^{2}}{U_{\mathrm{v}}^{2}\left[\left(c_{2}-c_{\mathrm{e}}\right)^{2}+c_{e}^{2} \Lambda^{2}\right]}=\operatorname{const}_{3} \frac{B_{\lambda: r}}{B_{\lambda r e l}} \text {. }
$$

Table I presents the measured values for the following quantities: the equivalence capacitance $c_{\mathrm{e}}$ both considering and neglecting $c_{3}$, the tangent of dielectric $\operatorname{loss} \operatorname{tg} \delta$, voltages on the reference and measurement cuvettes $U_{\text {ref }}$ and $U_{x}$ that were needed to achieve compensation of birefringence. Table I also gives the calculated capacitance of the measurement cuvette $c_{1}$, also both considering and neglecting $c_{3}$. The voltage on the cuvettes was determined by the kilovoltmeter of C-53 type. The measurements were carried out in the electric field with the frequency of $1 \mathrm{kHz}$. Two cuveties covered with a layer of dielectric were used. One cuvette with $35 \mathrm{~cm}^{2}$ electrodes was covered by fluoroplastic and contained a $2.5 \pm 0.1 \mathrm{~mm}$ wide liquid sample. The other cuvette with $74 \mathrm{~cm}^{2}$ electrodes was covered with mica and contained a $1.2 \pm 0.1 \mathrm{~mm}$ wide liquid sample. Further decrease in the width of the sample increased errors due to non-uniformity of the electric field in the cuvette. Perhaps, a different equivalence scheme can be designed to accommodate smaller samples. The temperature of the liquid samples was $24 \pm 1^{\circ} \mathrm{C}$, while $c_{3}=0.0662[\mathrm{p} \mathbf{F}]$.

TABLE I

Equivalence capacitance $c_{\mathrm{e}}$, both considering $c_{3}\left(c_{\mathrm{e}}\right)$ and neglecting $c_{3}\left(c_{\mathrm{e}}^{*}\right)$, tangent of dielectric losses $\operatorname{tg} \delta$ voltage on the reference $\left(U_{\text {ref }}\right)$ and measurement $\left(U_{x}\right)$ cuvettes as measured by the compensatory approach, together with the calculated values of the capacitance $c_{1}$ both considering $c_{3}\left(c_{1}\right)$ and neglecting $c_{3}\left(c_{1}^{*}\right)$.

\begin{tabular}{l|c|c|c|c|c|c|c}
\hline \multicolumn{1}{c|}{ Substance } & $c_{\mathrm{e}}[\mathrm{pF}]$ & $c_{\mathrm{e}}^{*}[\mathrm{pF}]$ & $\operatorname{tg} \delta$ & $U_{x}[\mathrm{kV}]$ & $U_{\text {ref }}[\mathrm{kV}]$ & $c_{1}[\mathrm{pF}]$ & $c_{1}^{*}[\mathrm{pF}]$ \\
\hline Air & 7.737 & 7.761 & 0.0142 & & & 12.481 & 12.456 \\
Benzene & 11.763 & 11.697 & 0.0139 & 3.00 & 2.90 & 28.366 & $28.231(5)$ \\
& & & & 3.00 & 1.30 & & \\
Tetrachloro- & 11.669 & 11.603 & 0.0138 & 3.00 & 1.32 & 27.820 & 27.691 \\
$\quad$ metan e & & & & & & & \\
1,4- Dioxane & 11.626 & 11.560 & 0.0142 & 3.00 & 1.30 & 27.573 & $27.446(5)$ \\
Toluene & 11.981 & $11.914(8)$ & 0.0140 & 2.00 & $2.68(5)$ & 29.836 & $29.534(6)$ \\
Propionic acid & 13.663 & 13.597 & 0.0159 & 2.00 & $2.94(1)$ & $42.874(3)$ & $42.570(5)$ \\
Chlorobenzene & 15.609 & 15.543 & 0.0178 & 1.00 & 2.95 & 70.579 & $69.858(5)$ \\
Pyridine & 17.808 & $17.741(8)$ & 0.0164 & 1.60 & 2.90 & 159.100 & $1.56 .598(1)$ \\
& & & & 3.00 & 0.354 & & \\
Nitrobenzene & 374.984 & - & 0.0174 & 0.200 & 3.00 & 2124.08 & - \\
Acetonitrile & 377.031 & - & 0.0168 & 0.500 & 2.88 & 2106.68 & - \\
Water & 419.084 & - & 0.0189 & 3.000 & 2.32 & 4358.67 & - \\
Acetic acid & 201.597 & - & 0.0180 & 0.600 & 3.00 & 359.62 & - \\
Sulfuric acid & 427.735 & - & 0.0191 & 2.900 & 2.96 & 5368.53 & - \\
& & & & & &
\end{tabular}




\section{TABLE II}

The cuvette constants const $t_{2}$ and const $_{3}$ calculated from reference measurements.

\begin{tabular}{c|l|c|c}
\hline \hline No. & \multicolumn{1}{|c|}{ Substance } & const $_{2}$ & const $_{3}$ \\
\hline 1 & Tetrachloromethane & 0.002770 & 0.002815 \\
2 & Benzene & 0.002747 & 0.002784 \\
3 & Pyridine & 0.002748 & 0.002832
\end{tabular}

TABLE III

Comparison with the literature data (Refs. $[37,38]$ ) of dielectric constants $\varepsilon$ and Kerr constants $B_{\lambda}$ measujed in this study.

\begin{tabular}{c|l|c|c|c|c}
\hline \hline No. & \multicolumn{1}{|c|}{ Substance } & $\varepsilon$ & $\begin{array}{c}B_{\lambda} \times 10^{15} \\
{\left[\mathrm{C}^{2} \mathrm{~m} \mathrm{~J}\right]}\end{array}$ & $\varepsilon[37]$ & $\begin{array}{c}B_{\lambda} \times 10^{-7} \\
{\left[\mathrm{C}^{2} \mathrm{~m} \mathrm{~J}^{-2}\right][38]}\end{array}$ \\
\hline 1 & Benzene & $2.27(3)$ & 3.92 & 2.275 & 3.92 \\
2 & Tetrachloromethane & $2.22(3)$ & - & 2.23 & 0.797 \\
3 & $1,4-$ Dioxane & $2.20(3)$ & 0.766 & 2.21 & 0.751 \\
4 & Toluene & $2.37(1)$ & 8.01 & 2.37 & 7.95 \\
5 & Propionic acid & $3.41(7)$ & 15.4 & 3.44 & 15.7 \\
6 & Chlorobenzene & $5.60(8)$ & $127 .(8)$ & 5.69 & 132.5 \\
7 & Pyridine & 12.57 & $222 .(4)$ & 13 & 219.2 \\
8 & Nitrobenzene & 37.21 & $358(6)$ & 36 & $3643-3974$ \\
9 & Acetonitrile & 36.9 & $53(1)$ & 37.5 & 519 \\
& & & 519 & & \\
10 & Water & 76.5 & 41.9 & 80 & $25.3-38.4$ \\
11 & Sulfuric acid & 93.5 & 110 & 100 & - \\
12 & Acetic acid & 6.29 & 42.2 & 6.30 & 44.0
\end{tabular}

The errors in the measured values of $c_{\mathrm{e}}$ were within 1 in the last significant digit. The constants that enter Eqs. (21) and (22) were determined by filling out both the reference and measurement cuvettes with the same reference liquids. The values of these constants for the $2.5 \mathrm{~mm}$ cuvetite are given in Table II. These values were calculated with and without taking account of $C_{3}$ based on measurements with carbon tetrachloricle, benzene, and pyridine. $B_{\lambda}$ of carbon tetrachloride used in the reference cuvette was taken equal to $0.797 \times 10^{-15} \mathrm{C}^{2} \mathrm{~m} \mathrm{~J}^{-2}$. Table III presents a comparison of the results obtained from the measurements in the $2.5 \mathrm{~mm}$ cuvette with the literature data. The differences in the Kerr constants between our data and the literature are no more than $5 \%$.

The cylindrical porcelain cuvette was used to measure $B_{\lambda}$ of nitrobenzene, which was purified by electro-dialysis [39]. The cuvette was suspended with capron 
threads in the middle of an air filled capacitor. The distance between the electrodes of the capacitor was $50 \mathrm{~mm}$. In order to achieve a uniform electric field inside the cuvette, the dimensions of the electrodes were much greater than the dimensions of the cuvetie. The cylindrical cuvette was sealed from the sides by glass, which was attached to the cylinder with a gelatin glue. The electric field applied to the electrodes of the air filled capacitor was $1 \mathrm{kFz}$ in frequency and $2 \mathrm{kV}$ in amplitude. Assuming that the dielectric permittivity of porcelain is $\varepsilon_{2}=6$, the strength of the electric field inside the sample was of the order of $32 \mathrm{~V} / \mathrm{cm}$. The constant of Eq. (19) as well as $B_{\lambda}$ of nitrobenzene weré calculated from the two measurements. First, the reference cuvette was filled with carbon tetrachloricle, second, the reference cuvette was filled with benzene. The Kerr constant of nitrobenzene that we obtained from the measurements in the cylindrical cuvette with isolated electrodes equaled $3200 \times 10^{-15} \mathrm{C}^{2} \mathrm{~m} \mathrm{~J}^{-2}$.

\section{Conclusion}

The analysis of the data obtained in this paper allows us to conclude that contactless capacitance measurements using low-frequency altemating electric currents lead to reliable and accurate information on the Kerr effect in liquid systems that are characterized by a wide range of electric conductivities.

\section{References}

[1] K. Rajagopal, T.A.P. Rao, B. Viswanathan, J. Phys. Soc. Jpn. 67, 658 (1998).

[2] J.M. Neto, A.B. Villaverde, J. Phys., Condens. Matter 8, 2791 (1996).

[3] J.M. Neto, E. Munin, A.B. Villaverde, Meas. Sci. Technol. 2, 1026 (1991).

[4] P. Peterson, A. Gavrieldes, E. Sakurada, J. Appl. Phys. 68, 446 (1990).

[5] C.G. Le F'evre, R.J.W. Le Fevre, in: Physical Methods of Chemistry, Ed. R.J.W Le Fevre. Vol. 1. Pt. IIIC. Interscience, New York 1972, p. 261.

[6] V.V. Prezhdo, M.V. Khashchina. V.A. Zamkov, Electro-Optical Investigation in Physics and Chemistry. Vyshcha. Schkola, Kharkov 1982, p. 152 (in Russian).

[7] C.J.F. Böttcher. C.O. van Belle, P. Bordewijk, A. Rip, Theory of Electric Polarization, Vol. 1, 2ndl ed., Elsevier, Amsterdam 1973, p. 377; C.J.F. Böttcher, C.O. va.n Belle, P. Bordewijk, in: Theory of Electric Polarization, Ed. C.J.F. Böttcher, Vol. 2, 2nd ed., Elsevier, Amsterdam 1978, p. 356.

[8] J.H. Williams, Chem. Phys. Lett. 147, 585 (1988).

[9] E.C. Cassidy, A.N. Cones, J. Res. Natl. Bur. Stand. 736, 5 (1969).

[10] G. Janmair, R. Coehlo, C.R. Acad. Sci., Paris 260, 1365 (1965).

[11] K. Tsujy, H. Watanabe, J. Chem. Phys. 66, 1343 (1977).

[12] K. Kikuchi, G. Honda, H. Watanabe, J. Phys. E, Sci. Instruments 21, 568 (1988).

[13] M. Pauthenier, J. Phys. Rad. 2, 181 (1921).

[14] Y. Chen, W.H. Orttung, J. Phys. Chem. 76, 216 (1972).

[15] R.K. Khanna, E. Dempsey, G.P. Jones, J. Phys. Lett. 53, 542 (1978).

[16] R.K. Khanna, P.K. Sharma, Czech. J. Phys. 38, 345 (1988).

[17] J.O. Ellis, J.P. Llewellin, J. Phys. E 10, 1249 (1977). 
[18] D.E. de Laney, S. Krause, Macromolecules 9, 455 (1977).

[19] A. Dobek, A. Pathowski, D. Labuda, J. Phys. D., Appl. Phys. 9, 121 (1976).

[20] J.C. Barnego, B. Roux, D. Herbade, Ber. Bunsenges. Phys. Chem. 80, 246 (1976).

[21] R. Tammer, W. Hutter, Chem. Phys. 146, 155 (1990).

[22] M. Paillette, J. Chimie Phys. 65, 1629 (1968).

[23] S. Kielich, Acta Phys. Pol. 30, 683 (1966).

[24] Z. Błaszczak, Acta Phys. Pol. A 71, 601 (1987).

[25] Z. Błaszczak, M. Farhond, J. Mol. Liquids 68, 157 (1996).

[26] Z. Błaszczak, H. Malek, Actc Phys. Pol. A 69,621 (1986).

[27] Z. Błaszczak, M. Farhoud, J. Mol. Liquids 62, 209 (1994).

[28] V.V. Prezhdo, I.P. Krainov, in: Intermolecular Interaction and Electrical Properties of Molecules, Ed. V.V. Prezhdo, Osnova, Kharkov 1994, p. 240 (in Russian).

[29] M.V. Khashchina, V.S. Raff, V.A. Zamkov, Prib. Tekh. Eksp. N2, 200 (1975).

[30] F. Eme, Dielektricheskie Izmereniya, Khimiya, Moskva. 1967, p. 223.

[31] S.V. Usikov, Elektrometriya Zhidkostei, Khimiya, Leningrad 1974, p. 144.

[32] V.S. Gangnus, A.N. Sus, V.V. Berezin, Izv. Vyssh. Uchebn. Zaved. Fiz. 39 (1975).

[33] M.P. Tonkonogov, V.A. Veksler, K.Zh. Birzhanov, Izv. Vyssh. Uchebn. Zaved. Fiz. 73 (1975).

[34] V.A. Zamkov, Instrum. Exp. Tech. 20, 1182 (1977).

[35] E. Duran, Electrostatique, Masson et ( ${ }^{\circ}$, Paris 1966, p. 389.

[36] L.D. Goldschtein, N.V. Zernov, Electromagnetic Fields and Waves, Sovetskoie Ractio, Moskva 1971, p. 662 (in Russian).

[37] Ya.Yu. Akhadov, Dielectric Properties of Pure Liquids, Izd. Standartov, Moskva 1972, p. 411 (in Russian).

[38] A.N. Vereshchagin, Polarizability of Molecules, Nauka, Moskva 1980, p. 177 (in Russian).

[39] V.V. Prezhdo, I.V. Romanov, M.V. Khashchina, C.N. Bliznietsov, E.I. Mikhed'kina, Vestnik Kharkov. Politekhnich. Inst. $N^{\circ} 146$, Khimiya i Khim. Tekhnologiya Org. Veshchestv, 45 (1978). 